\title{
Search for Neutrinoless Double Beta Decay with the COBRA Experiment
}

\author{
Tobias Koettig* for the COBRA Collaboration \\ $\dagger$ \\ TU Dortmund, Germany \\ E-mail: tobias.koettig@tu-dortmund.de
}

\begin{abstract}
Neutrinoless Double Beta decay is regarded to be the golden channel to answer the important question of the Dirac- or Majorana nature of the neutrino. First large scale experiments of this type are currently under commissioning. Should they succeed, experiments confirming their results are crucial. If not, approaches with a higher sensitivity are necessary. The COBRA project offers several promising and unique approaches to solve these challenging tasks.
\end{abstract}

35th International Conference of High Energy Physics - ICHEP2010,

July 22-28, 2010

Paris France

*Speaker.

$\dagger$ We want to thank the LNGS laboratory and the Deutsche Forschungsgemeinschaft for their support and the Z. He group of the University of Michigan for the possibility of operating their Polaris detector system in the COBRA R\&D set-up. 


\section{Neutrinoless Double Beta Decay}

Despite decades of intensive research, important properties of the neutrino such as its rest mass could not be determined yet. It is also the only fundamental particle that may be its own anti-particle, i.e. a Majorana particle. Observation of neutrinoless double beta decay $(0 v \beta \beta)$ can clarify both important questions at once. But due to the expected half-life of more than $10^{25}$ years it is an ambitious challenge to experimental physics .

Currently first experiments are under commissioning that will be able to probe this magnitude of half-lives [1] . If they succeed, it will be important to verify the results with other experiments using different isotopes and different approaches. If they do not succeed, again, new approaches will be required.

\section{The COBRA Experiment}

COBRA has the necessary properties to be an excellent candidate for such a successive experiment. The idea is to use CdZnTe room-temperature semiconductor detectors [2]]. CdZnTe contains several double beta decay candidate isotopes, among them also $\beta^{+}$emitters and two of the most promising isotopes, ${ }^{130} \mathrm{Te}$ and ${ }^{116} \mathrm{Cd} .{ }^{116} \mathrm{Cd}$ has a decay energy of $2809 \mathrm{keV}$ that lies well above the naturally occurring gamma background. It is a very good candidate for the observation of $0 v \beta \beta$ due to its good matrix element and large phase space.

The current COBRA R\&D low background test set-up is situated in the Italian underground laboratory LNGS. An overburden of 1400 meters of rock coverage reduces the cosmic ray flux by about six orders of magnitude. The test set-up is shielded with boron loaded polyethylene, more than two tons of lead and a core of ultra radio-pure electrolytic copper. With this test set-up a background level of less than 8 counts $/ \mathrm{keV} / \mathrm{kg} / \mathrm{yr}$ was achieved. With the results of current in-depth Monte-Carlo studies the shielding, especially regarding a large scale experiment, can be improved significantly.

CdZnTe semiconductor detectors have received more and more interest in recent years. Consequently, there has been major progress with these detectors. CdZnTe detectors are operated mainly with two readout techniques. The Co-Planar Grid (CPG) technology was developed specially for CdZnTe [3]. With this readout approach energy resolutions better than $2 \%$ FWHM at $662 \mathrm{keV}$ can be achieved and such detectors are commercially available. Even with cost efficient low resolution detectors resolutions of less than 2\% FWHM at $2615 \mathrm{keV}$ have been achieved at the COBRA low background test set-up. With this test set-up half-life limits above $10^{20}$ years for several isotopes and decay modes have been determined with CPG detectors [ [7. The current LNGS CPG set-up can house up to 64 of the $1 \mathrm{~cm}^{3}$ CPG detectors. For a large scale experiment one concept is an array consisting of 64000 such detectors with a total mass of $420 \mathrm{~kg}$. The advantage of an array is the high granularity. This will allow for coincidence studies that can be used to further reduce background or to search for decays to excited states. To achieve an even more effective background suppression and a maximum detection efficiency, the operation of CdZnTe CPG detectors in liquid scintillator is currently under investigation.

Operation of CdZnTe as pixelated detectors is also possible. When using pixelated detectors, COBRA is the only experiment that will have the opportunity to operate a kind of solid state TPC. 
Thus the unique combination of the large advantages of a "source equals detector" approach with the possibility of particle identification via particle track reconstruction is possible. In addition to in-depth Monte Carlo studies including charge transport also for the first time ever three different pixelated detector types have been operated in an ultra-low background environment. COBRA is investigating two types of pixelated detectors.

The first type are large volume detectors with a comparatively large pixel size of about $2 \mathrm{~mm}$. Two detector systems of this type have been operated in the low background R\&D test set-up. The first system was kindly provided by the Z. He group of the University of Michigan. It is a $2 \times 2 \times 1.5 \mathrm{~cm}^{3}$ detector with $11 \times 11$ pixels. Additionally to the xy-information from the hit pixels also information on the interaction depth is available. Even though this system was not optimised for low background operation, a cut on double beta like events that are likely to deposit their energy in at most two neighbouring pixels led to a background of 3.5 counts $/ \mathrm{keV} / \mathrm{kg} / \mathrm{yr}$ in the region of interest for ${ }^{116} \mathrm{Cd}$ from $2.7 \mathrm{MeV}$ to $2.9 \mathrm{MeV}$. By making an additional fiducial cut excluding the edges of the detector all events could be excluded in the ROI. Even though these cuts slightly reduce the detection efficiency for $0 v \beta \beta$ events and the sensitive detector volume, the demonstrated background reduction is impressive and shows the veto power of pixelated detectors. Also with the second large volume pixel detector, the first COBRA owned prototype, a $2 \times 2 \times 1 \mathrm{~cm}^{3}$ detector with $8 \times 8$ pixels, a low background level of 9 counts $/ \mathrm{keV} / \mathrm{kg} / \mathrm{yr}$ in the ROI for single pixel events was achieved.

The third pixelated detector type investigated by COBRA is the Timepix detector developed by the Medipix Collaboration. These detectors have a thickness of several mm's. With these thinner detectors much smaller pixel sizes in the order of $100 \mu \mathrm{m}$ are producible. These pixel sizes allow for event tracking and particle recognition. $\alpha \mathrm{s}, \beta \mathrm{s}$ and muons are clearly distinguishable. Also a discrimination of beta and double beta events can be achieved with good efficiency [5]. A detector with a pixel pitch of $110 \mu \mathrm{m}$ is currently running in the LNGS test set-up. Operating pixelated detectors in liquid scintillators may also be possible and will certainly be explored.

Taking all these innovative and promising possibilities of operating CdZnTe detectors into account shows the large potential of COBRA as a next generation experiment for the search for neutrinoless double beta decay.

\section{References}

[1] A. Giuliani, Acta Phys. Polon. B 41 (2010) 1447.

[2] K. Zuber, Phys. Lett. B 519 (2009) 1.

[3] P. N. Luke, IEEE Trans. Nucl. Sci. 42, (1995) 207.

[4] J. V. Dawson et al., Phys. Rev. C 80 (2009) 025502.

[5] T. R. Bloxham and M. Freer, Nucl. Instrum. Meth. A 572 (2007) 722. 